\title{
Megatrials for Bronchodilators in Chronic Obstructive Pulmonary Disease (COPD) Treatment: Time to Reflect
}

Wouter D. van Dijk, MD, Lisette van den Bemt, PhD, and Chris van Weel, MD, PhD

Introduction: Chronic obstructive pulmonary disease (COPD) is an important cause of morbidity and mortality worldwide. Although (long-acting) bronchodilators are used to relieve symptoms, the impact of bronchodilators on COPD mortality remains an unresolved issue. Our aim was to explore the results and the interpretations of the results of studies of bronchodilator treatment from high-impact COPD trials.

Methods: We searched PubMed and Embase for primary publications of randomized controlled trials with more than 1000 participants with COPD and that studied the effectiveness of long-acting bronchodilator treatment. We assessed population characteristics, primary outcomes, focus of outcomes, and possible bias from concomitant pulmonary medication.

Results: We retrieved 5 primary publications of large trials. Participants tended to be patients with rather severe COPD who were cared for at a hospital. Only half of the primary outcomes were statistically significant. Reports tended to focus on statically significant outcomes and not necessarily on primary outcomes or outcomes of the whole study population. The relevance of study outcomes was rarely discussed.

Discussion: The rather small effects of bronchodilators in a COPD population that is not representative for daily care, together with the tendency of relying on statistical rather than clinical significance, hampers translation to the large number of patients with COPD in the community. (J Am Board Fam Med 2013;26:221-224.)

Keywords: Bronchodilators, Chronic Disease, Chronic Obstructive Pulmonary Disease (COPD), Pharmacotherapy, Respiratory Tract Diseases

Chronic obstructive pulmonary disease (COPD) is a progressive chronic disease characterized by not fully reversible airflow obstruction. It is one of the most important causes of morbidity and mortality worldwide, directly related to cigarette smoking. Indeed, cessation of cigarette smoking is the single-most efficient intervention to prevent both disease develop-

This article was externally peer reviewed.

Submitted 23 December 2011; revised 23 March 2012; accepted 26 March 2012.

From the Department of Primary and Community Care, Radboud University Nijmegen Medical Centre, Nijmegen, the Netherlands.

Funding: none.

Conflict of interest: none declared.

Corresponding author: Wouter D. van Dijk, Department of Primary and Community Care, Radboud University Nijmegen Medical Centre, Internal Postal code 117, P.O. Box 9101, 6500HB, Nijmegen, The Netherlands (E-mail: w. vandijk@aios.umcn.nl). ment and progression. ${ }^{1,2}$ In addition, (long-acting) bronchodilators are used to relieve symptoms. An unresolved question is the impact of bronchodilators on COPD mortality, in part by attenuation of pulmonary function decline and exacerbations, independent from desired symptom relief. The last decade, a number of large studies on the effectiveness of longacting bronchodilators received extensive attention in leading medical journals. Calverley observed that "ensuring that these expensive studies are done objectively to the highest standard is an important goal". ${ }^{3}$ For that reason, the quality of these large trials, their external validity, and what they add to the current clinical practice, are of importance. We systematically reviewed the results and the interpretations of these results of megatrials on long-acting bronchodilators in COPD patients that were published in high impact journals. 


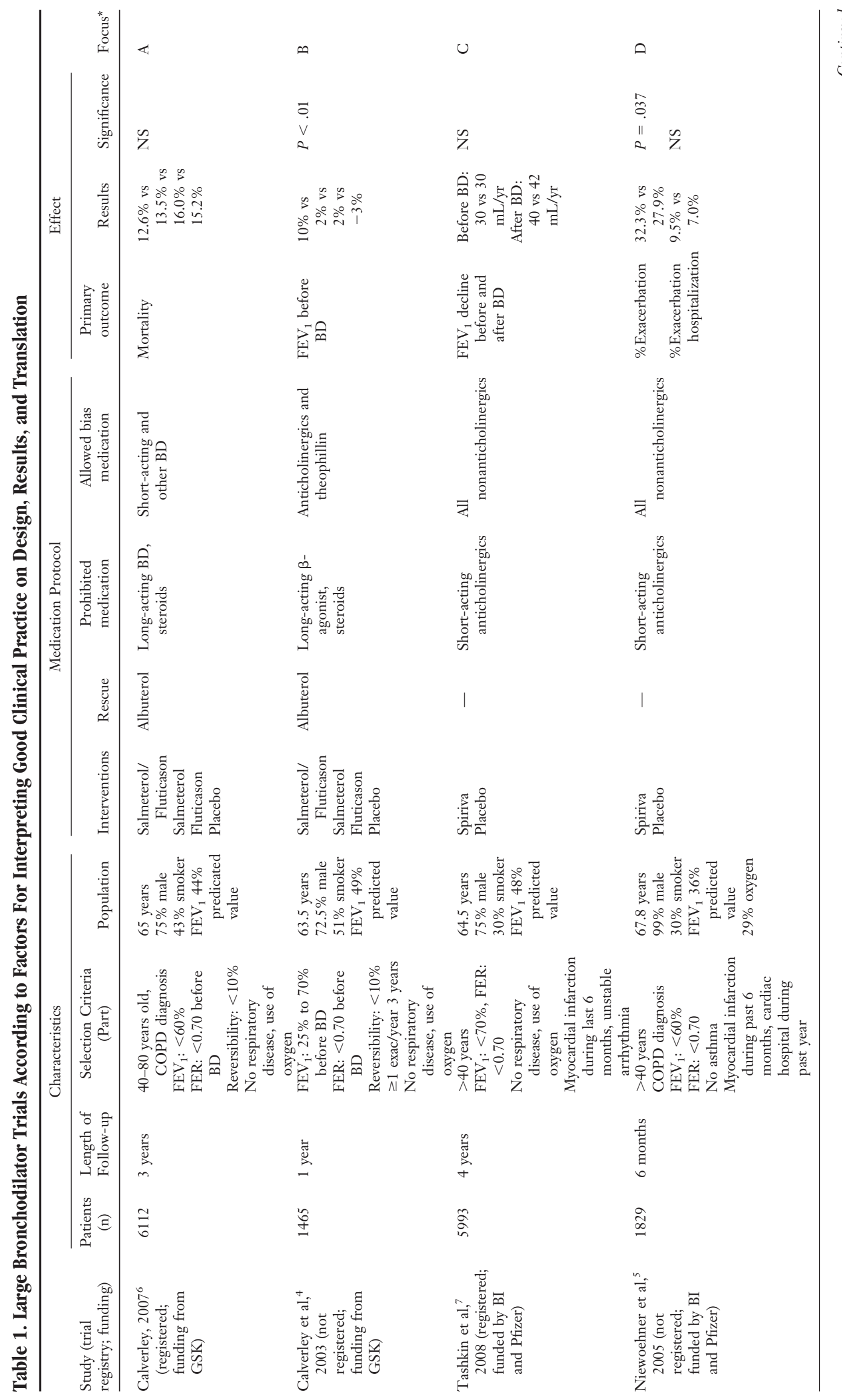




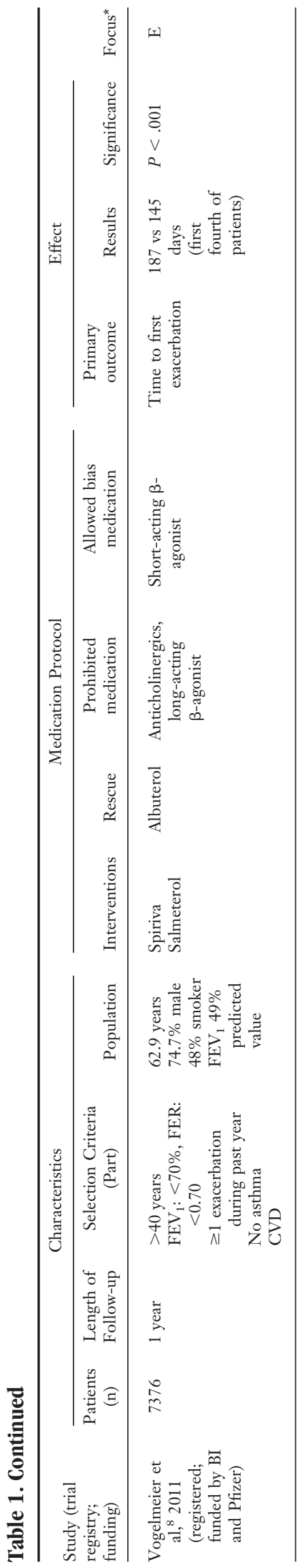

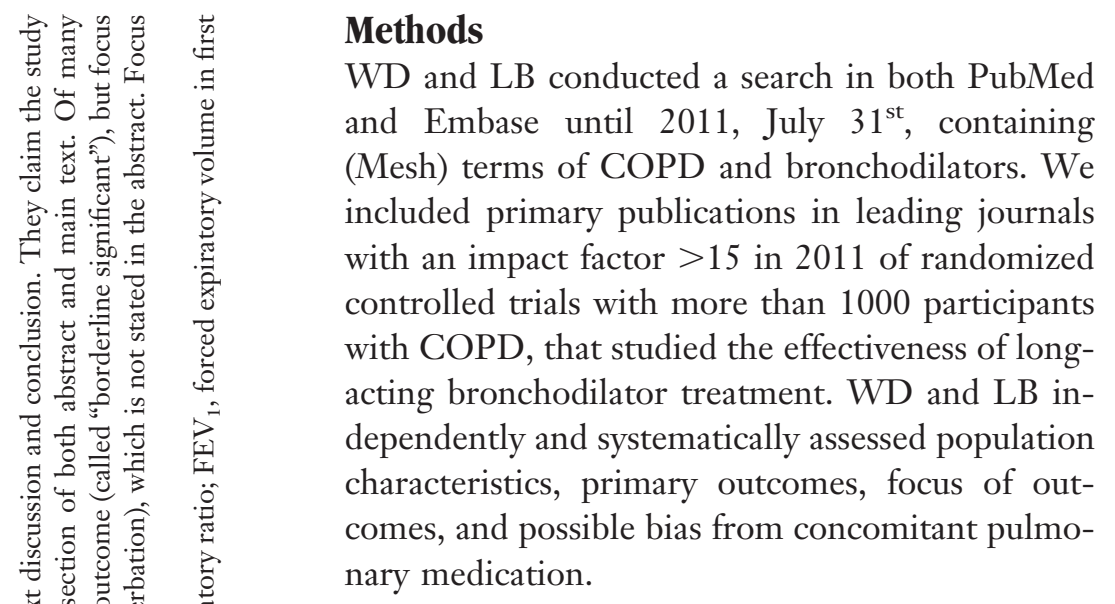

\section{Results}

We retrieved five primary publications of large trials on bronchodilator effect in COPD patients, including 1465 to 7376 patients with a mean follow-up between 6 and 48 months (Table 1). ${ }^{4-8}$ Mean COPD severity as measured by percentage of predicted forced expiratory volumes ranged from $36 \%$ to $49 \%$ at baseline. The mean proportion of males was $77 \%$. All studies included patients with at least 10 pack-years smoking history only, and patients with co-prevalence of asthma were excluded. Certain types of pulmonary co-medication were allowed during all studies, but none of the analyses were adjusted for these co-medications during follow-up. Table 1 also summarizes the various effects of bronchodilators on primary outcomes. Primary outcomes were mortality, ${ }^{6}$ exacerbations ${ }^{5,8}$ and lung function decline. ${ }^{4,7}$ Although all studies report positive outcomes for the study medication of interest in general, only three of six primary outcomes of the studies were statistically significant. ${ }^{4,5}$ Clinical significance was discussed in two trials only. ${ }^{4,5}$ The statistically significant primary outcomes include prebronchodilator $\mathrm{FEV}_{1}$ change (post-bronchodilator $\mathrm{FEV}_{1}$ was not statistically significant in this study), ${ }^{4}$ percentage of patients with exacerbations (the other primary outcome: number of hospitalizations for exacerbations, did not statistically differ significantly between groups), ${ }^{5}$ and time to exacerbations (a statistically significant modified population of analyses). ${ }^{8}$ Studies focused on statistically significant secondary outcomes, ${ }^{4,7}$ and statistically significant subgroup analyses. $^{7}$ Although two studies acknowledged statistically non-significant results for the primary outcome they focused on its beneficial effect, ${ }^{6,7}$ and on the secondary outcome. ${ }^{6}$ One study did not 
always correctly stated the population key characteristics, ${ }^{8}$ whereas another used pre-bronchodilator values as primary outcome. ${ }^{4}$

\section{Discussion}

Despite the positive tone in the reports of large trials on long-acting bronchodilator therapy in COPD patients, only half of the primary outcomes were statistically significant. Next, reports tend to focus on statically significant outcomes and not necessarily on primary outcomes or outcomes of the whole study population.

Compared with combining results of smaller rigorous trials into meta-analyses, megatrials could provide a small advantage on minimizing confounding by change. ${ }^{9}$ However, since large trials increase their participant numbers by reducing protocol rigidness, bias can be introduced that weakens causative interpretations. ${ }^{10}$ For instance, in these COPD megatrials, various co-medications were allowed during the study without proper adjustments for it in the analyses. On the other hand, decreased rigidness may provide a generalization of results in daily practice, but only if the study population is representative of the target population to which its results will be applied. ${ }^{9}$ Moreover, the clinical relevance of the rather small effects in a possibly biased COPD population that is not representative for daily care should be debated, in particular as meta-analyses rate these trials on their patient numbers mostly.

Most patients with COPD are treated in the community, while the selection of patients for large trials is biased toward referred, hospital cared patients. This, together with the tendency of relying on statistical rather than clinical significance, hampers translation to the large number of patients with COPD in the community. Independent from symptom relief, we would therefore plea for some precaution on the customary prescription of longacting bronchodilators for the COPD population at large.

\section{References}

1. Anthonisen NR, Connett JE, Kiley JP, et al. Effects of smoking intervention and the use of an inhaled anticholinergic bronchodilator on the rate of decline of FEV1. The Lung Health Study. JAMA 1994;272: 1497-505.

2. Kuller LH, Ockene JK, Meilahn E, Wentworth DN, Svendsen KH, Neaton JD. Cigarette smoking and mortality. MRFIT Research Group. Prev Med 1991; 20:638-54.

3. Calverley PM, Rennard SI. What have we learned from large drug treatment trials in COPD? Lancet 2007;370:774-85.

4. Calverley P, Pauwels R, Vestbo J, et al. Combined salmeterol and fluticasone in the treatment of chronic obstructive pulmonary disease: a randomised controlled trial. Lancet 2003;361:449-56.

5. Niewoehner DE, Rice K, Cote C, et al. Prevention of exacerbations of chronic obstructive pulmonary disease with tiotropium, a once-daily inhaled anticholinergic bronchodilator: a randomized trial. Ann Intern Med 2005;143:317-26.

6. Calverley PM, Anderson JA, Celli B, et al. Salmeterol and fluticasone propionate and survival in chronic obstructive pulmonary disease. N Engl J Med 2007;356:775-89.

7. Tashkin DP, Celli B, Senn S, et al. A 4-year trial of tiotropium in chronic obstructive pulmonary disease. N Engl J Med 2008;359:1543-54.

8. Vogelmeier C, Hederer B, Glaab T, et al. Tiotropium versus salmeterol for the prevention of exacerbations of COPD. N Engl J Med 2011;364:1093103.

9. Shrier I, Platt RW, Steele RJ. Mega-trials vs. metaanalysis: precision vs. heterogeneity? Contemp Clinic Trials 2007;28:324-8.

10. Charlton BG. Megatrials are based on a methodological mistake. Br J Gen Pract 1996;46:429-31. 